\title{
EMBARAZO EN ADOLESCENTES DE 11 A 15 AÑOS
}

\author{
Dr. Alberto Duarte-Contreras, M.D.*
}

El embarazo aumenta de manera "epidémica" en las adolescentes especialmente en las de clase económica menos favorecida; "brota como un volcán que despierta" debido a la pubertad más temprana, a los matrimonios cada vez a menor edad, a la "explosión de la cópula", a la "nueva libertad sexual" y al número de adolescentes que son aproximadamente el cincuenta por ciento de la población femenina.

Ellas y sus productos corren un grave riesgo durante el proceso del embarazo y del parto, riesgo que aumenta entre más jóvenes sean. EI desarrollo general insuficiente, la falta de equilibrio del sistema endocrino, la. tensión emocional lógica en el primer embarazo, la dieta deficiente y la falta de atención médica prenatal adecuada, hacen que se presenten graves problemas inherentes a la obstetricia de la adolescente tales como la toxemia, el aborto, la anemia ferropénica, el trabajo prolongado, la desproporción feto pélvica por falta de desarrollo de la pelvis materna, los niños de bajo peso al nacer y la alta mortalidad perinatal; motivos éstos que nos obligan a considerar al embarazo en adolescentes como un embarazo en alto riesgo $(1,3,4,17$, 27,32 ).

La situación empeora en adolescentess solteras pues ellas afrontan pro- blemas síquicos y sociales sobreagregados a todos aquellos de índole puramente orgánica. Si son estudiantes deben abandonar la escuela antes de estar debidamente capacitadas para ganarse la vida y se verán forzosamente inclinadas a depender de la ayuda que puedan ofrecerles los organismos de asistencia y bienestar social; u optarán por contraer matrimonio precipitadamente con muy pocas posibilidades de éxito; o se lanzarán en busca del aborto provocado con toda la pléyade de peligros y consecuencias; y no faltarán quienes desesperadas contemplen el suicidio como la única solución a su embarazo y lleguen a intentarlo y aún a consumarlo. Después del parto tanto la madre, soltera casi siempre, como el hijo pueden presentar graves problemas sicológicos y de adaptación social de muy difícil solución que dejan, en la mayoría de los casos, una marca definitiva.

\section{I - Incidencia}

De las 33.425 pacientes atendidas en el Servicio de Maternidad del Hospital San Juan de Dios, de Cúcuta (26.731 por parto y 6.694 por aborto) en el lapso 1960-1971 encontramos 492 gestantes con edad de 11 a

* Del Departamento Gineco Obstétrico del Hospital San Juan de Dios. Cúcuta, Colombia. 
15 años $(1,5 \%)$ o sea 1 por cada 66 pacientes. Incidencia baja si la comparamos con la encontrada por Gómez en Bogotá $8 \%$ (15) y por Castellanos en Caracas 20,6\% (7). Dife- rencia que abedece al medio ambiente aún reducido de nuestra ciudad donde las jovencitas no encuentran ni los peligros ni las oportunidades propias de la metrópoli.

EMBARAZO EN ADOLESCENTES DE 11 A 15 AÑOS. INCIDENCIA POR AÑO Y POR GRUPOS DE EDAD

\begin{tabular}{|c|c|c|c|c|c|c|c|c|}
\hline \multirow[b]{2}{*}{ Año } & Total & \multirow[b]{2}{*}{11} & \multirow{2}{*}{$\begin{array}{c}\text { Edad } \\
12\end{array}$} & \multicolumn{2}{|c|}{ adolescentes } & \multirow[b]{2}{*}{15} & \multirow{2}{*}{$\begin{array}{l}\text { Total } \\
\text { adoles- } \\
\text { centes }\end{array}$} & \multirow{2}{*}{$\begin{array}{c}\text { Porcentaje } \\
\text { adolescente } \\
\text { por año }\end{array}$} \\
\hline & Partos y Abortos & & & 13 & 14 & & & \\
\hline 1960 & 1.220 & & & & 3 & 4 & 7 & 0,5 \\
\hline 1961 & 1.782 & & & & 2 & 7 & 9 & 0,5 \\
\hline 1962 & 2.070 & & & & 5 & 10 & 15 & 0,7 \\
\hline 1963 & 2.244 & & & & 2 & 14 & 16 & 0,7 \\
\hline 1964 & 1.883 & & & 1 & & 18 & 19 & 1,0 \\
\hline 1965 & 2.518 & & & & 8 & 19 & 27 & 1,0 \\
\hline 1966 & 3.030 & & & 2 & 10 & 33 & 45 & 1,4 \\
\hline 1967 & 2.583 & 1 & & 1 & 7 & 41 & 50 & 1,9 \\
\hline 1968 & 3.521 & & & & 12 & 51 & 63 & 1,6 \\
\hline 1969 & 3.649 & & 1 & 2 & 15 & 50 & 68 & 1,8 \\
\hline 1970 & 4.252 & & 1 & 1 & 13 & 59 & 74 & 1,7 \\
\hline 1971 & 4.673 & & & 3 & 19 & 77 & 99 & 2,1 \\
\hline TOTAL & 33.425 & 1 & 2 & 10 & 96 & 383 & 492 & \\
\hline
\end{tabular}

Al observar la incidencia por año y por grupos de edad vemos un franco aumento en todas las edades a partir de 1966.

De los 445.915 partos y abortos registrados oficialmente en Colombia en 1969, setecientos cuarenta y uno $(0,29 \%)$ corresponden a jóvenes de 10 a 14 años (12).

\section{II - Edad}

2

EMBARAZO EN ADOLESCENTES

DE 10 A 14 AÑOS DE EDAD

\begin{tabular}{|c|c|c|c|}
\hline Edad & $\mathrm{N}^{0}$ & de Pacientes & $\%$ \\
\hline 11 & & 1 & 0,2 \\
\hline 12 & & 2 & 0,4 \\
\hline 13 & & 10 & 2,3 \\
\hline 14 & & 96 & 19,5 \\
\hline 15 & & 383 & 77,6 \\
\hline TOTAL & & 492 & \\
\hline
\end{tabular}

El grupo más numeroso corresponde a los 15 años $(77,6 \%)$. Tenemos pocos embarazos en jóvenes de 11 , 12 y 13 años $(0,3 \%$ del total de piacientes atendidas y $2,9 \%$ del grupo de adolescentes estudiadas). La edad promedio fue de 14,7 similar a la encontrada por Van der Ahe en un grupo de adolescentes americanas de 10 a 15 años en ciudad con densidad cle población igual a la nuestra (29).

\section{III - Menarca}

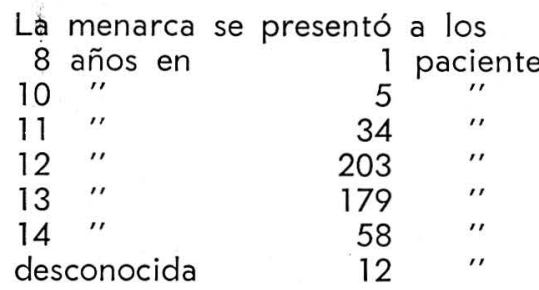

La edad promedio fue de 12 años, cifra similar a la encontrada por Cas- 
tellanos en Caracas $(7,10,11)$. Ninguna de nuestras pacientes fue embarazada antes de la aparición de su primera menstruación. Se admite que mientras más temprana sea la menarca, aumenta con mayor rapidez la tasa de desarrollo pélvico (18). En nuestro medio la niña inicia un crecimiento acelerado entre los 9 y los 11 años, y la menarca se presenta ordinariamente un año después de la aparición de esta tasa máxima de crecimiento; cuando llega la menarca, cualquiera sea la edad cronológica, la tasa de maduración esquelética se sitúa entre los 13 y medio y los 14 años. El mecanismo que rige este rápido crecimiento premenárquico es desconocido, si bien parece depender de factores genéticos primordialmente $(14,16,19)$.

\section{IV - Gestación y paridad}

\section{3}

EMBARAZO EN ADOLESCENTES DE 11 A 15 AÑOS. - GESTACION

\begin{tabular}{|c|c|c|c|c|c|}
\hline Edad & G I & G II & $\begin{array}{l}\text { de Paci } \\
\text { G III }\end{array}$ & G IV & Total \\
\hline 11 & 1 & & & & 1 \\
\hline 12 & 2 & & & & 2 \\
\hline 13 & 9 & 1 & & & 10 \\
\hline 14 & 92 & 4 & & & 96 \\
\hline 15 & 370 & 7 & 3 & 3 & 383 \\
\hline TOTAL & 474 & 12 & 3 & 3 & 492 \\
\hline
\end{tabular}

Cuatrocientas setenta y cuatro adolescentes $(96,2 \%)$ cursaban su primera gestación, doce eran secundigestas $(2,6 \%)$, tres eran $G$ III $(0,6 \%)$ y tres G IV.

De las 492 adolescentes, 452 eran primíparas $(96,2 \%)$. Si tenemos en cuenta que durante los once años del presente estudio se atendieron 7.101 primíparas en nuestro Servicio, el grupo de primíparas adolescentes representa el $5,8 \%$, cifra intermedia en-
4

EMBARAZO EN ADOLESCENTES

DE 11 A 15 AÑOS. - GESTA Y PARIDAD

\begin{tabular}{|c|c|c|c|c|}
\hline Gesta & y Paridad & $N^{0}$ de & Pacientes & $\%$ \\
\hline G I & Primíparas & & 434 & 96,2 \\
\hline \multirow[t]{2}{*}{ G II } & Primíparas & & 9 & 1,9 \\
\hline & Secundíparas & & 3 & 0,7 \\
\hline \multirow[t]{2}{*}{ G III } & Primíparas & & 2 & 0,4 \\
\hline & Secundíparas & & 1 & 0,2 \\
\hline \multirow[t]{2}{*}{ G IV } & Primíparas & & 1 & 0,2 \\
\hline & Secundíparas & & 2 & 0,4 \\
\hline
\end{tabular}

tre la encontrada por Gómez en el Instituto Materno Infantil de Bogotá $(2,37 \%)$ y la observada por Hassan en el Booth Memorian Hospital $(8,3 \%)(15,18)$. Las dos G IV secundíparas eran prostitutas que habían tenido su primer embarazo a los 12 años.

\section{$\checkmark$ - Estado civil}

Hay un gran predominio de adolescentes solteras $(70,8 \%)$, cifra cercana a la encontrada en Bogotá $73,7 \%$ y en Caracas $70,7 \%$ para citar datos de latitudes semejantes ( 7 , $11,15)$ y similar a la observada por investigadores de países mejor desarrollados (20).

\section{5}

EN:BARAZO EN ADOLESCENTES

DE 11 A 15 AÑOS. - ESTADO CIVIL

\begin{tabular}{ccc}
\hline Edad & Solteras & Casadas \\
\hline 11 & 1 & \\
12 & 2 & \\
13 & 10 & \\
14 & 60 & 36 \\
15 & 276 & 107 \\
TOTAL & 349 & 143 \\
$\%$ & 70,8 & 29,2 \\
\hline
\end{tabular}

Todas las pacientes de 11, 12 y 13 años eran solteras. La morbilidad materna, la mortalidad perinatal, el parto prematuro y el bajo peso de los 
recién nacidos de adolescentes jóvenes se han relacionado a la ilegitimidad, como también a los matrimonios “obligados a cañón de escopeta", concepto que compartimos basados en los hallazgos de nuestro estudio. Sin embargo, autores como Ballard, Parmelee y Wallace afirman que el embarazo en una soltera adolescente no predispone de por sí a la prematuridad sino que es simplemente un síntoma potencial en el cual juegan papel componentes sociales, emocionales y físicos $(4,25,30,31)$.

\section{VI - Control prenatal}

Cincuenta y cinco pacientes tuvieron control prenatal: 23 desde el primer trimestre, 19 a partir del segundo y 13 en el tercero. Solamente 14 tuvieron cuatro o más consultas prenatales; el descuido en la asistencia prenatal se reflejará claramente en la aparición de la toxemia y en la prematurez. Cuatrocientas treinta y siete tuvieron la primera entrevista obstétrica cuando llegaron al Servicio para la atención del aborto o del parto; ciento tres llegaron con un trabajo superior a las 12 horas. De las 40 que finalizaron su gestación en aborto, ninguna tuvo control prenatal.

El grupo de casadas solicitó en mayor proporción este servicio que el grupo de solteras: 36 c a sadas $(25,1 \%)$ y 19 solteras $(5,4 \%)$. Ninguna de las pacientes de 11, $12 \circ 13$ años, solteras todas, asistió a control prenatal. Esta falta de control obedece a un sinnúmero de factores: ignorancia, familiares, sociales, de conveniencia, emocionales como también a la ligereza con que de ordinario se toman a esta edad situaciones de tánta trascendencia.

\section{VII - Evolución del embarazo}

A. - ABORTO.
Las estadísticas del Departamento Administrativo Nacional de Estadística, DANE, registran para Colombia 82.068 abortos en 1969, de los cuales $108(0,13 \%)$ corresponden a pacientes de 10 a 14 años (12).

De los 6.694 abortos atendidos en nuestro Hospital, $40(0,59 \%)$ pertenecen a adolescentes de 11 a 15 años correspondiendo la mayor incidencia al grupo de los 15 años. En 18 $(3,8 \%)$ encontramos antecedente obstétrico (ver Cuadro 4). No tuvimos embarazo ectópico ni mola hidatiforme. Todos los abortos fueron confirmados por estudio histopatológico. No pudimos comprobar abortos provocados. La proporción de los abortos $(8,1 \%)$ fué muy inferior a la de los partos $(91,9 \%)$. Esta incidencia similar a la encontrada en estudios realizados en países económicamente bien desarrollados, variará profundamente en el futuro a medida que se vaya legalizando en ellos el aborto. No encontramos datos sobre uso de métodos anticonceptivos. La totalidad de estas pacientes Ilegaron con aborto en curso o con aborto incompleto; todo ello demuestra falta de educación sexual y desconocimiento de la conducta humana en la vida familiar (3).

La incidencia de abortos de nuestro Servicio es muy alta en términos generales $(20,4 \%)$ debido al gran número de abortos provocados; por.centaje que se aprecia visiblemente elevado al compararlo con el de abortos en adolescentes $(8,1 \%)$, grupo en el cual no encontramos aborto provocado.

Si analizamos por grupos de edad las pacientes estudiadas por varios investigadores, observamos que el porcentaje de abortos espontáneos disminuye a medida que aumenta la edad, lo que es apenas lógico, pues 
6

EMBARAZO EN ADOLESCENTES DE 11 A 15 AÑOS. - EVOLUCION DEL EMBARAZO

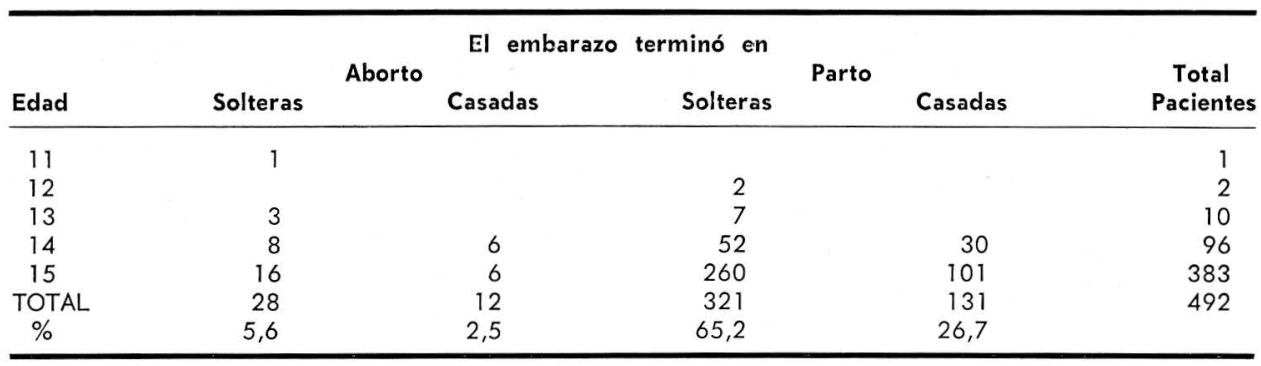

al aumentar la edad la adolescente adquiere un mayor desarrollo y una mayor aptitud para la procreación. $\mathrm{Si}$ bien la edad muy temprana puede intervenir como causante de aborto, es preciso, en estudio de adolescentes, desechar la idea de que la edad es el factor etiológico primordial y abordar la investigación de otras posibles causas tales como anomalías uterinas, zoonosis, hemopatías, alteraciones genéticas, etc.

Compartimos con Ballard (4) el concepto de que la adolescente sexualmente activa necesita desesperadamente ayuda para diferir el embarazo hasta que haya edad y situación más oportuna. En esta forma se podrían impedir los embarazos no deseados y se obtendría un control muy significativo al problema del aborto y específicamente al del aborto provocado. Mas paradógicamente quienes más necesitan regular la natalidad, las adolescentes muy jóvenes, son quienes más desconocen los métodos anticonceptivos y quienes más difícil acceso tienen a los centros de estudio de control natal. Sin embargo, como lo anoto Osofsky (24) "a causa de la escasez de programas de educación sexual para adolescentes que nunca se han embarazado, todavía no se dispone de datos que puedan indicar los efectos de estos ser- vicios sobre el número y la proporción de nacimientos extramaritales en adolescentes".

Debemos recordar con perplejidad que en ciertas oportunidades, muy pocas por fortuna y en esferas socioeconómicas altas, es la madre de la adolescente quien pide el aborto como única solución al embarazo de su hija.

B. - TOXEMIA.

\section{7}

EMBARAZO EN ADOLESCENTES

DE 11 A 15 AÑOS. - TOXEMIA

\begin{tabular}{|c|c|c|c|c|c|c|}
\hline \multirow[b]{2}{*}{ Eclad } & \multicolumn{3}{|c|}{ Preeclampsia } & \multicolumn{3}{|c|}{ Eclampsia } \\
\hline & $\mathrm{N}^{0}$ & Pacientes & $\%$ & $N^{o}$ & pacientes & $\%$ \\
\hline 11 & & & & & & \\
\hline 12 & & & & & & \\
\hline 13 & & 2 & 0,6 & & 3 & 0,6 \\
\hline 15 & & 15 & 3,6 & & 16 & 3,5 \\
\hline 14 & & 12 & 2,6 & & 2 & 0,4 \\
\hline TCTAL & & 30 & 6,5 & & 21 & 4,5 \\
\hline
\end{tabular}

Encontramos 51 toxémicas (11\%) todas por encima de los 13 años: 30 preeclámpticas $(6,5 \%)$ y 21 eclámpticas $(4,5 \%)$, cifra bastante baja si la comparamos con la de otros autores tales como Gómez (19,35\% (15), Castellanos 16,7\% (7) y Claman $32 \%$ (8). Esta cifra del $11 \%$ es muy alta para nosotros si tenemos en 
cuenta que la incidencia de nuestro Servicio es tan sólo del $6 \%$.

La toxemia fue más frecuente en las solteras: 35 solteras, $60,3 \%$. La eclampsia apreció preferencialmente en el grupo de los 15 años. Sólamente 13 preeclámpticas y 4 eclámpticas habían tenido control prenatal. De las 17 toxémicas que concurrieron al control prenatal, tres presentaron aumento exagerado de peso, y en el grupo de las que no tuvieron control prenatal ocho tuvieron un marcado aumento de peso.

De las 51 toxémicas, tres dieron mortinatos; de los 48 productos nacidos vivos, dos murieron en los primeros días. Los hijos de las adolescentes toxémicas suelen tener buen pronóstico y la mortalidad es relativamente baja; ello obedece a que la toxemia empieza al final del embarazo y no está sobreañadida a enfermedad hipertensiva crónica como sucede en madres de mayor edad (4).

Los factores que contribuyen a la aparición de la toxemia en gestantes muy jóvenes son primordialmente la falta de desarrollo y de balance del sistema endocrino, la tensión emocional, la asistencia prenatal insuficiente y la inmadurez del organismo materno en relación con los cambios que debe sufrir durante el embarazo $(18,21)$. La desnutrición puede guardar también relación con la toxemia (4) : la mayoría de la gestantes jóvenes son desnutridas por sus bajas condiciones económicas o por su creciente deseo de alcanzar y conservar figuras delgadas a costa de dietas absurdas y mal balanceadas. Las gestantes muy jóvenes están destinadas a tener un promedio de embarazos superior al de aquellas que comienzan a concebir a mayor edad. La toxemia, así sea leve, las lleva con paso apresurado e inexorable hacia un daño re- nal, y hacia la enfermedad hipertensiva desde los primeros años de fertilidad; el ataque toxémico temprano al sistema cardio vásculo-renal, algunas veces repetido, puede contribuir también a la aparición de la toxemia o al aumento en gravedad en posteriores embarazos y aún a trastornos vasculares cardíacos y renales crónicos en edad más avanzada.

\section{C. - ANEMIA.}

Sólo en 65 historias clínicas encontramos los datos de hemoglobina y hematocrito. Las cifras de hemoglobina fueron de 8 a 11 gramos con un promedio de 10 y las de hematocrito de $32 \%$ a $38 \%$ con un promedio de $36 \%$. La mayoría de los investigadores han observado anemia en embarazadas adolescentes con cifras de hemoglobina inferiores a los $10 \mathrm{gr}$. en el $20 \%$ (1). El requerimiento de hierro durante la adolescencia es bastante alto pues debe satisfacer las necesidades del crecimiento, reponer las pérdidas sanguíneas de cualquier naturaleza y conservar la reserva utilizable cle este mineral en posible gestación; pero existe aumento en la capacidad de absorción del hierro en la época del crecimiento y particularmente cuando hay un embarazo concomitante (21). En nuestro grupo de estudio no encontramos anemia de tipo ferropénico que pudiésemos atribuir directamente al embarazo: las bajas cifras de hemoglobina y de hematocrito que encontramos parecen tener como causa primordial el poliparasitismo intestinal y el deficiente estado nutricional de las pacientes.

D. - PARTO.

De los 363.847 partos registraclos en Colombia por el DANE en 1969, seiscientos treinta y tres $(0,16 \%)$ corresponden a jóvenes de 10 a 14 años (12). De los 26.731 partos aten- 
didos en nuestro Hospital en los 11 años del presente estudio, 452 se realizaron en adolescentes de 11 a 15 años, uno por cada 59 partos $(1,7 \%)$; datos éstos muy parecidos a los suministrados por Clark quien observó 540 embarazos en adolescentes americanas de 10 a 15 años al estudiar 28.600 partos atendidos en 11 años en el Hospital Freedmen's de Washington (6). No encontramos partos múltiples.

8

EMBARAZO EN ADOLESCENTES

DE 11 A 15 AÑOS. - PARTO PREMATURO

\begin{tabular}{lccr}
\hline Edad & Solteras & Casadas & Total \\
\hline 11 & & & \\
12 & & & \\
13 & 5 & & 5 \\
14 & 36 & 6 & 42 \\
15 & 40 & 15 & 55 \\
TOTAL & 81 & 21 & 102 \\
$\%$ & 79,4 & 20,6 & \\
\hline
\end{tabular}

Ciento dos adolescentes (26,5\%) terminaron su gestación en parto prematuro: cifra acorde con las encontradas por todos los autores que hemos consultado. El $79,4 \%$ de los partos prematuros se realizó en adolescentes solteras.

\section{9}

EMBARAZO EN ADOLESCENTES

DE 11 A 15 AÑOS. - PARTO A TERMINO

\begin{tabular}{lccr}
\hline Edad & Solteras & Casadas & Total \\
\hline 11 & & & \\
12 & 2 & & 2 \\
13 & 2 & & 2 \\
14 & 28 & 12 & 40 \\
15 & 220 & 86 & 306 \\
TOTAL & 252 & 98 & 350 \\
$\%$ & 70,5 & 29,5 & \\
\hline
\end{tabular}

De los 350 partos a término, 252 corresponden a adolescentes solteras $(70,5 \%)$. Si tenemos en cuenta que
321 solteras terminaron su gestación en parto, el $31,7 \%$ del total de solteras tuvo parto prematuro, en tanto que en el grupo de casadas se presentó tan sólo en el $16 \%$.

\section{E. - TRABAJO DE PARTO.}

La duración del trabajo de parto fue de 12 horas en promedio, cifra comparable con la de un grupo de primíparas mayores de 20 años de nuestro Servicio ( 11 horas). Cabe anotar que 103 adolescentes llegaron a la Maternidad con un trabajo superior a las 12 horas. El trabajo más corto, 4 horas, lo realizaron cinco pacientes de 13 y 14 años; el más prolongado, 28 horas, se observó en dos pacientes de 15 años. Todos los trabajos mayores de 20 horas, 27 en total, se presentaron en pacientes de 15 años. Veinticuatro partos fueron conducidos con ocitócicos.

La duración del trabajo en adolescentes muy jóvenes suele ser o muy corta o muy prolongada y está en razón directa a la incompatibilidad feto pélvica (3). Semens al hacer hincapié en los trabajos demasiado cortos que se observan en adolescentes jóvenes dice: "cuidado con la adolescente que tiene cuello uterino de mujer que ha parido!" (26).

La presentación cefálica la encontramos en 329 partos (95\%), la de pelvis en $12(2,6 \%)$ y fue desconocida en $11(2,4 \%)$.

El parto fue espontáneo en 359 pacientes $(79,5 \%)$, e intervenido en 93 $(20,5 \%)$. El fórceps se empleó en 36 $(7,9 \%)$ : en 23 por demora en expulsivo, en dos como profiláctico y en uno para rotar una variedad posterior. El Vacuum o Extractor al vacío lo usamos en 33 pacientes $(7,3 \%)$ para acelerar el período expulsivo. 
La operación cesárea se practicó en 24 pacientes (5,3\%): por desproporción feto pélvica en 9, por sufrimiento fetal en 4, por pelvis en primigesta en 3, por procidencia del cordón en 2, por desprendimiento prematuro de placenta normoinserta en 2 , por placenta previa en 2 y por inercia uterina en 2.

La incidencia de cesárea en nuestras adolescentes $(5,3 \%)$ es inferior al índice general de nuestro Servicio $(8,9 \%)$. El índice de cesárea para pacientes muy jóvenes varía según los autores y va desde 0,4 hasta $8 \%$ (20). Parecería raro que habiendo 46 productos con peso superior a los 3.500 gramos sólamente se hubiesen practicado nueve cesáreas por desproporción feto pélvica; pero el desarrollo de la pelvis femenina en la pubertad se caracteriza por el crecimiento rápido que ocurre con tal impulso que la porción ósea del conducto reproductor puede ser adecuada para el paso y el nacimiento de un niño a término en menos de un año después de la pubertad (23). Cuando transcurre un tiempo prudencial entre la fecha de la menarca y la concepción, el parto se efectúa fácilmen- te por vía vaginal siempre que no medien otras complicaciones, y por lo tanto en estas condiciones la frecuencia de pelvis estrecha como indicación para la operación cesárea es muy similar en la parturienta adolescente y en la mujer adulta (6).

Practicamos episiotomía a 201 pacientes; creemos que debe hacerse a todas las primíparas de esta edad, y de manera generosa en muchos casos, dado el desarrollo incompleto del periné; evitaremos así los grandes desgarros causantes de serios problemas en el futuro obstétrico y ginecológico, y sobre todo en el futuro sexual de la paciente.

Observamos 41 desgarros de periné: doce grado I $(6,6 \%)$, veinte grado II $(4,4 \%)$ y nueve grado III $(1,9 \%)$, desgarros que se hubieran evitado si hubiésemos practicado la episiotomía.

Cuando el trabajo progresa normalmente y el parto es vaginal, los procedimientos utilizados, los requisitos y la atención son idénticos a los que se practican en mujeres de mayor edad (18).

EMBARAZO EN ADOLESCENTES DE 11 A 15 AÑOS. - TEST DE APGAR

\begin{tabular}{|c|c|c|c|c|c|c|c|c|c|c|c|}
\hline \multirow[b]{2}{*}{ Edad } & \multirow[b]{2}{*}{2} & \multirow[b]{2}{*}{3} & \multirow[b]{2}{*}{4} & \multirow[b]{2}{*}{5} & \multicolumn{3}{|c|}{$\begin{array}{l}\text { Número de paciente } \\
\text { Con Test de Apgar }\end{array}$} & \multirow[b]{2}{*}{9} & \multirow[b]{2}{*}{10} & \multirow[b]{2}{*}{ Sin dato } & \multirow[b]{2}{*}{ Total } \\
\hline & & & & & 6 & 7 & 8 & & & & \\
\hline 11 & & & & & & & & & & & D \\
\hline 12 & & & & 1 & & & 1 & & & & 2 \\
\hline 13 & 1 & & 2 & 2 & 1 & & 1 & & & & 7 \\
\hline 14 & 1 & 4 & 2 & 4 & 19 & 14 & 21 & 5 & 8 & 5 & 83 \\
\hline 15 & 1 & 2 & 13 & 26 & 32 & 37 & 104 & 88 & 28 & 8 & 339 \\
\hline TOTAL & 3 & 7 & 17 & 33 & 52 & 51 & 127 & 93 & 36 & 13 & 431 \\
\hline$\%$ & 0,7 & 1,6 & 3,9 & 7,6 & 12 & 11,8 & 29,4 & 21,5 & 8,3 & 3,2 & \\
\hline
\end{tabular}

\section{VIII - Recién nacidos}

En los 452 partos se obtuvieron 431 productos vivos $(95,3 \%)$ y 21 mortinatos $(4,7 \%)$. La edad no influye sobre la calificación de Apgar. EI $86,2 \%$ de los productos tuvieron un Apgar entre 6 y 10 , pero con un ma- 
yor número de productos ubicados en la calificación de $8(29,4 \%)$. Todos los test inferiores a $6(13,8 \%)$ correspondieron a productos inmaduros o nacidos en trabajos descuidados. Desafortunadamente en ninguna de las historias aparece la valoración del Apgar a los 5 minutos; en relación con predicción de mortalidad antes de dos días tiene valor la calificación a los 5 minutos que la tomada al minuto de nacer.

\section{IX - Prematurez}

El índice general de productos de bajo peso 0 prematuros en nuestro Servicio fue de $9,2 \%$ en 1963 (13) y bajó a $8,6 \%$ en 1968 (9). El índice de prematurez de los productos de nuestras adolescentes $(22,5 \%)$ es semejante al encontrado por Gómez en Bogotá $(22,9 \%)$ pero muy elevado si lo comparamos con los hallazgos de Castellanos (16\%) y de Clark $(13,94 \%)(16,6,7)$.

El producto de menor peso, 730 gr., correspondió a una primípara de 14 años, y el de mayor peso, 4.270 gr. a una secundípara de 15 años. Setenta y tres productos eran hijos de madres solteras.

Si buscamos el índice de prematurez por grupos de edad encontramos:

3 productos en madres de 13 años $(42,7 \%)$

28 productos en madres de 14 años 74 productos en madres de 15 años $(34,1 \%)$

$(20,9 \%)$, o sea la prematurez es sensiblemente más elevada entre menor sea la edad de la adolescente. En las últimas etapas de la vida reproductiva también se aprecia un aumento en la prematurez, pero estudios tan serios como los de Briggs (5),

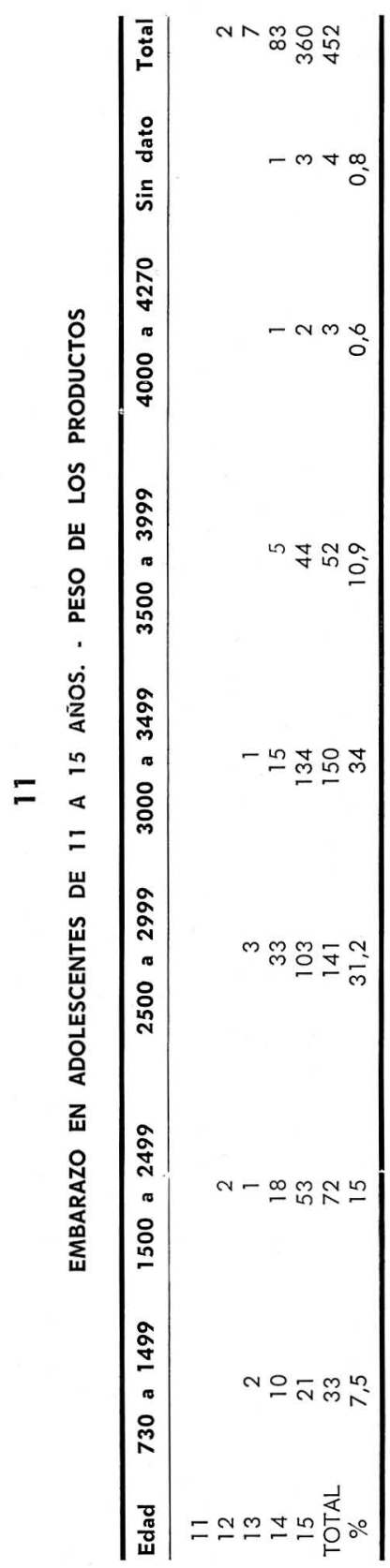


Claman (8) y Hassan (18) han observado que el riesgo de tener hijos prematuros es el doble en mujeres menores de 15 años que el de cualquier otra gestante que se encuentre en los límites superiores de edad. Nuestro alto índice de prematurez bien puede tener relación con la falta de control prenatal, con la carencia alimenticia y con la ilegitimidad, aseveración que por demás hacen todos los autores. Según Bishop, citado por Balard (4), se ha comprobado que la desnutrición sí tiene un efecto ominoso sobre el aumento de frecuencia de productos de bajo peso a más de todas las demás causas enunciadas.

\section{$X$ - Mortalidad perinatal}

Hubo 21 mortinatos $(4,7 \%)$ y 11 mortineonatos $(2,4 \%)$. Los 21 mortinatos pertenecieron tres a pacientes de 13 años, tres a pacientes de 14 años y 15 a pacientes de 15 años; 16 eran hijos de solteras. La muerte obedeció a desprendimiento prema- turo de placenta normoinserta en 2 casos, a prolapso del cordón en 3 , a eclampsia en 3 y a sufrimiento fetial en 13.

Los once mortineonatos tenían un Apgar al minuto por debajo de 5; uno de ellos había nacido de madre de 13 años, seis de madre de 14 años y cuatro de madre de 15 años. Siete eran hijos de solteras. La causa cle su muerte fue: anoxia del recién nacido en 4 , hemorragia intracraneana en 2, bronconeumonía en 2, membrana hialina en 1, hemorragia pulmonar en 1 e inmadurez respiratoria en 1 .

Nuestra mortalidad perin a t a I $(7,1 \%)$ es muy elevada a todas luces si la comparamos con la del Instituto Materno Infantil de Bogotá $5,3 \%$ (15) y con la de la Maternidad Concepción Palacios de Caracas $4,1 \%$ (7).

\section{XI - Placentas}

12

emibarazo EN ADOLESCENTES DE 11 A 15 AÑOS. - PESO de LAS PLACENTAS

\begin{tabular}{|c|c|c|c|c|c|c|c|}
\hline Edad & 200 a 299 & 300 a 399 & 400 a 499 & 500 a 599 & 600 a 699 & 700 a 760 & Total \\
\hline 11 & & & & & & & \\
\hline 12 & & & 2 & & & & 2 \\
\hline 13 & 1 & 2 & 1 & & & & 4 \\
\hline 14 & 3 & 8 & 44 & 8 & & & 63 \\
\hline 15 & 2 & 8 & 89 & 95 & 2 & 2 & 198 \\
\hline TOTAL & 6 & 18 & 136 & 103 & 2 & 2 & 267 \\
\hline$\%$ & 2,2 & 6,6 & 5,1 & 38,8 & 0,7 & 0,7 & \\
\hline
\end{tabular}

El peso promedio de las placentas fue de $480 \mathrm{gr} .$, inferior al de doscientas placentas de pacientes entre $20 \mathrm{y}$ 30 años que fue de 510 gramos. Aun cuando el número de placentas estudiadas es reducido apreciamos que el $59,8 \%$ tiene un peso inferior a los 500 gramos y de éstas el $8,8 \%$ están por debajo de los 400 gramos. Las placentas de menor peso no corres- pondían a pacientes de menor edad pero sí guardaban relación con los productos de bajo peso y con las gestaciones por debajo de las 34 semanas.

Se acepta que el peso normal de la placenta es de 500 gramos y que hay correlación entre su peso y el de los recién nacidos (2); también que el 
peso de la placenta está de acuerdo con la edad de la gestación. Las placentas de bajo peso son placentas inmaduras a las que se atribuye el síndrome de "insuficiencia placentaria".

Al estudio histopatológico de algunas de las placentas encontramos hematomas retroplacentarios, infartos placentarios, inserción excéntrica del cordón, trofoblasto atrófico, cambios hialinos, cambios fibrinoides, alteraciones todas que nos explican el bajo peso de los productos, el bajo índice de Apgar y la mortalidad de algunos recién nacidos.

\section{$X I 1$ - Malformaciones congénitas}

Las malformaciones congénitas fueron aparentes en cinco recién nacidos vivos $(1,1 \%)$ : polidactilia en 2 , ectopía testicular en 1, equino valgus en 1 y pie chapín en 1. Este índice de $1,1 \%$ es discretamente superior a la incidencia general de nuestro Servicio $0,97 \%$, pero muy inferior a la encontrada por Gómez en Bogotá $3 \%$ (15). Cuando se analizan grupos de varios millares de recién nacidos de adolescentes de muy temprana edad se observa que no hay tendencia a una mayor proporción de malformaciones congénitas que cuando se estudian recién nacidos de madres de mayor edad $(1,3,4,6,11,18)$.

\section{XIII - Hemorragia}

Ocho pacientes presentaron hemorragia: ods por desprendimiento prematuro de placenta normoinserta, dos por placenta previa y cuatro por inercia uterina en el puerperio inmediato. Cinco necesitaron transfusión sanguínea en cantidad de 500 a 1.000 cc.

\section{XIV - Puerperio}

Fue normal en 429 casos (94,9\%) y complicado en $23(5,1 \%)$. Las complicaciones fueron: dehiscencia de episiorrafía en 3 , endometritis en 8 , mastitis en 4, dehiscencia de herida quirúrgica en 3 , infección de herida quirúrgica sin dehiscencia en 3, e insuficiencia renal aguda en 2 . No tuvimos mortalidad materna.

\section{$X V$ - Conclusiones}

La adolescente muy joven presenta un significativo aumento de la morbilidad durante la gestación, el parto $y$ el puerperio.

Estadísticamente está comprobado que la adolescente casada o debidamente amparada tanto afectiva como socialmente presenta menos problemas que la adolescente embarazada soltera o desamparada.

Es de primordial importancia insistir sobre la necesidad del cuidado prenatal.

Toda adolescente embarazada debe recibir durante su gestación y puerperio el cuidado de un sicólogo experimentado, quien al mismo tiempo debe prestar su colaboración a los padres de la futura madre, casi siempre portadores de un trauma síquico que les puede conducir a actitudes erradas.

Es indispensable solicitar la colaboración de la familia para atenuar - resolver los problemas de la madre adolescente y de su hijo, problemas cuya solución depende del conocimiento y comprensión de sus causas, del amparo familiar, afectivo, económico, social y médico asistencial.

Es indispensable intensificar los programas de educación sexual a partir del hogar y de la escuela pero nos nace la inquietud de la falta de idoneidad de los padres de familia y aún de los educadores. No creemos 
que la liberación de la adolescente embarazada esté en el "aborto legalizado", muy al contrario, estamos convencidos de que él llevará a la desnaturalización del instinto más noble de toda mujer y a la pérdida del respeto a la vida ajena.

Las maniobras, procedimientos y requisitos para la atención del parto en adolescentes muy jóvenes son semejantes a las que se practican en la mujer adulta, pero requiere control solícito y responsable a más de buena dosis de tino para no desorientar el futuro obstétrico y sexual de las pacientes.

El embarazo en adolescentes muy jóvenes no debe considerarse simplemente como un embarazo de alto riesgo, sino ante todo como un problema social puesto que en más del $70 \%$ de los casos se realiza en solteras desamparadas e inexpertas. Entre nosotros este problema puede llegar a graves y trascendentales consecuencias dada la situación demográfica del país, que por demás es la que corre el mundo entero. Los siguientes datos corroboran nuestra afirmación (12):

1․ Total de mujeres en Colombia menores de 15 años:

\begin{tabular}{ccc}
\hline $\begin{array}{c}\text { Estimación a } \\
\mathbf{1 5} \text { de Julio de }\end{array}$ & $\begin{array}{c}\text { Total de } \\
\text { mujeres }\end{array}$ & $\begin{array}{c}\text { Mujeres de } \\
\mathbf{1} \mathbf{1 4} \text { años }\end{array}$ \\
\hline & & \\
1969 & $10^{\prime} 381.500$ & $4^{\prime} 712.450$ \\
1970 & $10^{\prime} 713.000$ & $4^{\prime} 862.900$ \\
1971 & $10^{\prime} 056.000$ & $5^{\prime} 018.600$ \\
\hline
\end{tabular}

2. El índice de aumento del total de población femenina en el país en base al censo de 1964 (este censo dio un total de 8'869.856, cifra que se toma como $100 \%$ ) es el siguiente:

\begin{tabular}{lc}
\hline Año & Indice de Aumento \\
\hline 1967 & 9,91 \\
1968 & 13,42 \\
1969 & 17,04 \\
\hline
\end{tabular}

3. La tasa de crecimiento geornétrico anual de mujeres en el país es de 32,7 por mil.

\section{$X V I$ - Resumen}

Se estudian 492 adolescentes $\mathrm{em}$ barazadas que tenían de 11 a 15 años de edad, todas del Servicio de Maternidad del Hospital San Juan de Dios, de Cúcuta, Colombia. El 70,8\% eran solteras. Se demuestra la influencia de la edad, del estado civil de solteras y de la falta de control prenatal sobre la mayor incidencia de toxemias, de partos prematuros, de productos de bajo peso y de mortalidad perinatal al comparar los promedios con los de grupos de mayor edad del Servicio. Las malformaciones congénitas tuvieron un índice de 1,1\% discretamente superior a la cifra de nuestra Maternidad. Se hacen comentarios sobre cada uno de los tópicos analizados y se dan conclusiones.

\section{Summary}

492 adolescent pregnant women, edged 11 to 15, were studied at the obstetric Service of the "Hospital San Juan de Dios" in Cúcuta, Colombia. The $70.8 \%$ were no married. The influence of age, civil stage and prenatal care on incidence of Toxemia $(11 \%)$, premature births $(26,5 \%)$. Low weig newborns $(22.5 \%)$, and High perinatal mortality $(7.1 \%)$ is comented in comparison with the promediums of eldery mothers in the same obstetric Service. The congenital malformations in $\mathrm{th}$ is $\mathrm{grup}$ $(1.1 \%)$ were lightly superior to the general promedium in our Service. On made comentaries and give conclutions about every one of the diferent analized topics. 


\section{XVII - BIBLIOGRAFIA}

1 AZNAR, R. and BENNETT, A. E. Pregnancy in the adolescent girl. Am. J. Obst. Gyn. 81: 934, 1961.

2 ABERNE, W. A. and DUNNILL, M. S. Cuantitative aspects of placental structure. J. Path. Bact. 91: 123, 1966.

3 BATTAGLIA, F. C., FRAZIER, T. M. and HELLEGERS, A. E. Obstetric and Pediatric complications of juvenile pregnancy. Pediatrics. 39: 902, 1963.

4 BALLARD, W. M. y GOLD, E. M. Aspectos médicos y de salud de la reproducción en la adolescencia. Clin. Obstet. y Ginec. 338, 1971.

5 BRIGGS, R. M., HARREN, R. R. and THOMPSON, W. B. Pregnancy in the young adolescent. Am. J. Obstet. Gynec. 84: 436, 1962.

6 CLARK, J. F. J. Obstetricia en adolescentes: deducciones obstétricas y ginecológicas. Clin. Obstet. Ginec. 1026, 1971.

7 CASTELLANOS, R., AGUERO, O., FRANCO, G. y MORENO, L. F. Estudio sobre mil madres adolescentes menores de 15 años. Rev. Obst. Gin. Venez. 29: 65, 1969.

8 CLAMAN, A. D. and BELL, H. M. Pregnancy in the very young teen-age. Am. J. Obstet. Gynec. 90: 350, 1964.

9 CORONEL, J. G., BUSTAMANTE, G. y URI$\mathrm{BE}, \mathrm{G}$. Prematuridad en nuestro medio. Pediatría. 10: 275, 1968.

10 CASTELLANOS, R., AGUERO, O., FRANCO, G. y ALLER, J. Estudio de la gestante adolescente. Rev. Obst. Gin. Venez. 10: 197, 1970.

11 CASTELLANOS, R., AGUERO, O., ALLER, J., FRANCO, C. Y NEUMAN, G. Problemas de la gestante de 15 y 16 años de edad. Rev. Obst. Gin. Venez. 32: 255, 1972.

12 Datos suministrados por el Departamento Administrativo Nacional de Estadística, DANE, en comunicación personal.

13 DUARTE-CONTRERAS, A., CORONEL, J. G. y BUSTAMANTE, G. Mortalidad perinatal en el Hospital San Juan de Dios de Cúcuta. Rev. Col. Obst. Gin. 15: 319, 1964.

14 DUARTE-CONTRERAS, A. . .isiología de las hormonas sexuales en la infancia. Rev. Col. Obst. Gin. 22: 385, 1971.
15 GOMEZ, J. A. Obstetricia en adolescentes. Rev. Col. Obst. Gin. 23: 353, 1972.

16 GOLFARB, A. F. Pubertad. Clin. Obst. Gin. 769, 1968.

17 HULKA, J. F. and SCHAAF, J. T. Obstetrics in adolescents: a controlled study of deliveries by mothres 15 years of age and under. Obstet. Gynec. 23: 678, 1964.

18 HASSAN, H. M. and FALLS, F. H. The young primipara. Am. J. Obstet. Gynec. 88: 256, 1964.

19 HARRIS, H. J. Problemas del crecimiento en el adolescente. Clin. Med. de Norte Am. 357, 1965.

20 ISRRAEL, S. L. and WONTERSZ, T. B. Teenage obstetrics. Am. J. Obstet. Gyn. 85: 659, 1963.

21 McGANITY, W. G. y cols. Relación entre la nutrición y el embarazo en la adolescencia. Clin. Obst. Gin. 367, 1971.

22 MONSEN, E. R., KUHN, I. N. and $\mathrm{FINCH}$ C. A. Iron status of menstruating women. Amer. J. Clin. Nutr. 20: 842, 1967.

23 MUSSIO, T. Primigravidas under age 14 Am. J. Obstet. Gyn. 84: 442, 1962.

24 OSOFSKY, H. J. Aspectos generales del embarazo extramarital en adolescentes. Clin. Obst. Gin. 442, 1971

25 PARMELEE, A. H. Jr. Prematurity and illegimacy. Am. J. Obstet. Gynec. 81: 81, 1961.

26 SEMMENS, J. P. Implication of teen-age pregnancy. Obstet. Gynec. 26: 77, 1965.

27 UTIAN, W. H. Obstetrical implications of pregnancy in primigravidas aged 16 years or less. Brit. Med. J. 2: 734, 1967.

28 VON DER AHE, C. The unwed teen-age mother. Am. J. Obstet. Gynec. 104: 279, 1969.

29 VON DER AHE. Problems in the management of illegitimate pregnancy. Am. J. Obstet. Gynec. 86: 607, 1963.

30 WALLACE, H. M. Teen-age pregnancy. Am. J. Obstet. Gynec. 92: 1125, 1965.

31 WALLACE, H. M. Factores relacionados con la mortalidad y morbilidad perinatales. Clin. Obst. Gin. 13, 1970.

32 ZACKER, J., ANDELMAN, S. L. and BAUER, $F$. The young adolescent as an obstetric risk. Am. J. Obstet. Gynec. 103: 305, 1969. 